\title{
Clinical Supervision Frameworks for Allied Health Professionals: A Systematic and Critical Review
}

Marcus Gardner

La Trobe University, mgardner@bendigohealth.org.au

Carol McKinstry

La Trobe University, c.mckinstry@latrobe.edu.au

Byron Perrin

La Trobe University, B.Perrin@latrobe.edu.au

Follow this and additional works at: https://nsuworks.nova.edu/ijahsp

Part of the Medicine and Health Sciences Commons

\section{Recommended Citation}

Gardner M, McKinstry C, Perrin B. Clinical Supervision Frameworks for Allied Health Professionals: A Systematic and Critical Review. The Internet Journal of Allied Health Sciences and Practice. 2021 Jan 01;19(3), Article 2.

This Systematic Review is brought to you for free and open access by the College of Health Care Sciences at NSUWorks. It has been accepted for inclusion in Internet Journal of Allied Health Sciences and Practice by an authorized editor of NSUWorks. For more information, please contact nsuworks@nova.edu. 


\title{
Clinical Supervision Frameworks for Allied Health Professionals: A Systematic and Critical Review
}

\begin{abstract}
Purpose: Clinical supervision is an important element of professional support for allied health professionals and contributes to the provision of safe, high quality patient care and health professional wellbeing. Structured clinical supervision frameworks have been recommended to improve access and effectiveness of clinical supervision for allied health professionals by providing practical guidance and increased consistency. However, there is limited evidence relating to the availability and quality of clinical supervision frameworks for allied health. Method: A systematic and critical review was conducted to identify and appraise clinical supervision frameworks for allied health. Included were peer-reviewed studies and grey literature documents, available in full text and written in English. Six databases and government and professional association websites were searched. The AGREE Health Systems Guidance (AGREE-HS) tool was used to appraise framework quality. Three researchers independently reviewed the frameworks and reached consensus on scores through discussion. AGREE-HS scores were analysed descriptively. Results: Twenty-six frameworks were appraised by the AGREE-HS including 7 peer-reviewed studies and 19 grey literature documents. Over half of all frameworks were from Australia, and the profession/s that they related to were most commonly allied health, social work, or psychology. The combined mean of the AGREE-HS final items scores for all studies/documents was $14.5(\mathrm{SD}=4.0)$ out of a possible score of 35. Frameworks published in peer-reviewed studies used more robust methods to inform their development than frameworks sourced from the grey literature. In contrast, grey literature frameworks were often more clearly outlined, succinct, practical, and flexible for stakeholders to implement. Conclusions: There are limited published frameworks available for allied health professionals, and the frameworks that do exist are generally of low quality. As a result, many existing frameworks may not provide the practical guidance required to improve clinical supervision practice and optimise the benefits of clinical supervision. It is recommended that future policy relating clinical supervision needs to focus on the development of common, evidence-based allied health clinical supervision frameworks. Future frameworks should be practically orientated and use robust methods and evaluation to inform their development and implementation.
\end{abstract}

\section{Author Bio(s)}

Marcus J. GardnerB App Sc (Pod), is a PhD candidate with La Trobe Rural Health School. He is a registered podiatrist and Manager of Allied Health Education at Bendigo Health.

Carol McKinstryPhD, M Hlth Sc, B App Sc (OT), Grad Cert in HE, is an Associate Professor and Head of the Department of Allied Health at La Trobe Rural Health School, Bendigo. She is a registered occupational therapist.

Byron Perrin PhD, M HIth Sc, B Pod, is the Head of the Department of Community Health at La Trobe Rural Health School, Bendigo. He is a registered podiatrist. 


\title{
Clinical supervision frameworks for allied health professionals: a systematic and critical review
}

\author{
Abstract \\ Purpose: Clinical supervision is an important element of professional support for allied health professionals and contributes to \\ the provision of safe, high quality patient care and health professional wellbeing. Structured clinical supervision frameworks \\ have been recommended to improve access and effectiveness of clinical supervision for allied health professionals by providing \\ practical guidance and increased consistency. However, there is limited evidence relating to the availability and quality of clinical \\ supervision frameworks for allied health. Method: A systematic and critical review was conducted to identify and appraise \\ clinical supervision frameworks for allied health. Included were peer-reviewed studies and grey literature documents, available in \\ full text and written in English. Six databases and government and professional association websites were searched. The \\ AGREE Health Systems Guidance (AGREE-HS) tool was used to appraise framework quality. Three researchers independently \\ reviewed the frameworks and reached consensus on scores through discussion. AGREE-HS scores were analysed \\ descriptively. Results: Twenty-six frameworks were appraised by the AGREE-HS including, 7 peer-reviewed studies and 19 \\ grey literature documents. Over half of all frameworks were from Australia and the profession/s that they related to were most \\ commonly allied health, social work or psychology. The combined mean of the AGREE-HS final items scores for all \\ studies/documents was 14.5 (SD = 4.0), out of a possible score of 35. Frameworks published in peer-reviewed studies used \\ more robust methods to inform their development than frameworks sourced from the grey literature. In contrast, grey literature \\ frameworks were often more clearly outlined, succinct, practical and flexible for stakeholders to implement. Conclusions: There \\ are limited published frameworks available for allied health professionals and the frameworks that do exist are generally of low \\ quality. As a result, many existing frameworks may not provide the practical guidance required to improve clinical supervision \\ practice and optimize the benefits of clinical supervision. It is recommended that future policy relating clinical supervision needs \\ to focus on the development of common, evidence-based allied health clinical supervision frameworks. Future frameworks \\ should be practically orientated and use robust methods and evaluation to inform their development and implementation.
}




\section{Background}

Allied health professionals make a significant contribution to health and social care systems, optimising the health and wellbeing of patients. 1,2 While there is no agreed definition of allied health across different settings and jurisdictions, professions often described as allied health include physiotherapy, occupational therapy, speech pathology, dietetics, podiatry and exercise physiology and, in the Australian context, social work and psychology. ${ }^{3-5}$ Clinical supervision is widely practised amongst allied health professions as a mechanism for clinical governance and to support professional wellbeing. ${ }^{6}$

Clinical supervision has been defined as "the formal provision, by approved supervisors, of a relationship-based education and training that is work-focused and which manages, supports, develops and evaluates the work of colleague/s". 7 p. 440 It is proposed that clinical supervision contributes to the provision of safe, high quality healthcare by promoting evidence-based practice, improving clinical reasoning and creating opportunities for reflection and feedback. ${ }^{6}$ Clinical supervision assists professional wellbeing by helping allied health professionals to manage the emotional demands of practice by providing a confidential space to discuss clinical issues and has been found to reduce professional isolation and burnout, particularly for rural allied health professionals..$^{6,8,9}$

Issues relating to the standards of clinical supervision for health professionals in the United Kingdom have been identified as a contributing factor to serious breaches of patient safety. ${ }^{10}$ In Australia, health system failures in recent years have highlighted the need for health services to ensure that there are appropriate systems and processes in place for effective clinical governance to ensure the delivery of safe and quality clinical care. ${ }^{11}$ Internationally, health systems are also undergoing significant change. For example in Australia, current changes include aged care reforms, ${ }^{12}$ the implementation of the National Disability Insurance Scheme, ${ }^{13}$ an increasing emphasis on reducing hospital lengths of stay, ${ }^{14}$ and the impact of the COVID-19 pandemic. 15,16 Significant healthcare change has been reported to negatively impact health professionals wellbeing and lead to feelings of constantly "having to do more with less". ${ }^{17}$ Clinical supervision has been highlighted as a factor to support allied health professionals maintain professional resilience during times of change. ${ }^{18}$

There are many definitions and interpretations of clinical supervision, with a lack of consensus across allied health professions regarding what clinical supervision is and how it should be implemented. ${ }^{19,20}$ Much of the historical basis for the practice of clinical supervision in allied health is derived from social work and psychology which has impacted on how clinical supervision is conceptualised and practised in the other allied health professions. ${ }^{10}$ There is relatively little evidence for best practice models of clinical supervision for allied health professions beyond social work and psychology. ${ }^{7}$

Although clinical supervision is well-accepted and widely practised amongst allied health professionals, there are inconsistent expectations for access to clinical supervision and processes, training and resources, to support best practice approaches.7,21 Issues relating to lack of access to clinical supervision have been highlighted in rural and regional settings where allied health professionals may be professionally and geographically isolated. ${ }^{22,23}$ There are also differences in the perceived effectiveness of clinical supervision across allied health professions, with a number of recent studies reporting that psychologists, social workers and occupational therapists perceived that their clinical supervision was effective while physiotherapists and dietitians reported lower levels of perceived effectiveness. ${ }^{24-26}$

A repeated recommendation of recent Australian research has been the need for practical, structured clinical supervision frameworks, specifically developed for allied health, to address inconsistencies and to improve the quality of clinical supervision for allied health.7,27-29 A number of clinical supervision frameworks have been developed to provide guidance to health professionals and health care organisations in the implementation and practice of clinical supervision. ${ }^{30-34}$ Clinical supervision frameworks described in the literature usually consist of numerous components, such as a conceptual "model" or "map" that helps guide practitioners by outlining parameters for the practice of clinical supervision. ${ }^{30}$ Other framework components may include a protocol or guideline that outlines the roles and responsibilities of supervisees, clinical supervisors and managers; recommendations for the evaluation and measurement of the framework's implementation; and tools and resources that can support clinical supervision practices and/or preparation and training of clinical supervisors. ${ }^{19,} 30,35$ These components are used to optimise the effectiveness of clinical supervision through informing practice and evaluation. ${ }^{30}$

While some government agencies and professional and regulatory bodies in Australia and the United Kingdom have recently developed clinical supervision frameworks or policies for allied health, ${ }^{32-34,36}$ these are limited to relatively few professional groups and jurisdictions therefore they are not available for a significant proportion of allied health professionals. Where clinical supervision frameworks are available, there is limited evidence relating to their quality and utilisation. ${ }^{30}$ It is unclear whether the clinical supervision frameworks available for allied health are providing guidance that will improve the quality of clinical supervision practice. 


\section{Aims}

The aim of this review was to examine the existing evidence for allied health clinical supervision frameworks, assess their quality and identify gaps. The objectives of this review were to 1 . Identify existing allied health clinical supervision frameworks for clinical supervision of allied health professionals, 2. Assess the quality of frameworks with an emphasis on their suitability to provide practical guidance to allied health professionals and health service managers employing allied health professionals, 3 . Make recommendations for the further development or implementation of existing frameworks or inform the development of future frameworks and 4 . Inform future policy directions relating to clinical supervision implementation.

\section{Methods}

\section{Search methods}

The review included peer-reviewed quantitative and qualitative studies of any research design. A detailed search strategy was developed prior to an initial search of electronic databases being undertaken on October 31, 2018 and a subsequent search on March 20 2020. The following search terms were used: (supervis* or clinical supervis* or professional supervis* or staff supervis*) and (model or framework or policy or guideline or manual or review or toolkit or implementation plan) and (allied health or physiotherap* or occupational therap* or social work* or speech pathology* or speech therap* or speech and language therap* or diet* or podiatr* or exercise physiolog* or psycholog*). Databases searched included the Cochrane Central Register of Controlled Trials (CENTRAL), MEDLINE (Ovid), Cumulative Index Nursing and Allied Health Literature (CINAHL) (EBSCO), EMBASE (Ovid), ProQuest (Nursing and Allied Health Database) and Psychlnfo. An example of a search strategy from the ProQuest database is provided in a supplementary file (see Supplementary file 1).

Clinical supervision frameworks developed by government or professional organisations documents are often located in the grey literature, therefore the grey literature was also searched. Using the search terms for the database search as a guide, a search of grey literature was conducted to identify clinical supervision frameworks published on organisation or government websites, while the Cochrane database and Google Scholar were included in the search.

\section{Eligibility criteria}

Eligibility criteria were established prior to the search of electronic databases and grey literature. To be eligible, studies/documents had to: be written in English, published in peer-reviewed journals or on government/organisational websites, available as full texts, published after 2000 , have a primary focus on clinical supervision and allied health, and describe a framework or model that could be used for practical guidance. The scope of the review is clinical supervision of qualified allied health professionals, rather than undergraduate students or supervision for higher degree by research students.

\section{Definitions}

The terms "framework", "model" and "theory" are inconsistently applied and often used interchangeably in the literature, ${ }^{35,37}$ including in research relating to clinical supervision. This review focused on frameworks that provide guidance and recommendations for the implementation of clinical supervision theory into practice, therefore definitions derived from the field of implementation science were used. Included were structured frameworks that could inform policies, decision making and judgments for the implementation of effective clinical supervision practice. Implementation frameworks have been described in research implementation science as "action process models". ${ }^{35}$ Process models specify steps (stages, phases) to guide the process of translating research into practice, including the implementation and use of research. An action process model provides practical guidance in the planning and execution of implementation endeavours and/or implementation strategies to facilitate implementation. ${ }^{35}$ The terms "model" and "framework" can both be used to describe the concept of an action process model. Alternate terms used to describe frameworks may include policy framework, health service guideline, manual, review, toolkit and implementation plan. ${ }^{38}$ Studies describing theories or conceptual models and frameworks were not eligible for appraisal.

\section{Identification of included papers}

The Preferred Reporting Items for Systematic Reviews and Meta-Analyses (PRISMA) guidelines were used as a reference for reporting this review. ${ }^{39}$ The initial screening of titles and abstracts of all located studies were conducted by one researcher and then full texts were retrieved for those not excluded at this stage. Full text studies and grey literature documents were then reviewed independently by two researchers against the inclusion criteria. Where there was disagreement on inclusion of studies, consensus was reached by discussion between the two reviewers, with a third reviewer involved where necessary. The reference lists of eligible studies were examined to identify potential studies that were not identified through the initial search process. 


\section{Method quality appraisal}

The Appraisal of Guidelines for Research and Evaluation - Health Systems (AGREE-HS) was used to appraise the quality of the identified frameworks. ${ }^{38}$ This tool was developed to assess the quality and usability of documents, providing guidance for health services and reported to be a usable, reliable and valid tool.41-44 The AGREE-HS was adapted from the AGREE-II tool which has been widely used for the appraisal and development of clinical guidelines. ${ }^{4,41}$

The AGREE-HS tool contains 5 items, each of which are rated on a 7-point Likert scale from lowest quality (1) to highest quality (7). The first item, Topic, refers to the how well the health system issue and its causes are described and the relevance of the guidance. Participants examines the composition of the team developing the framework and the management of team member and funding conflicts. The third item, Method, includes the use of systematic methods to consider evidence to inform the framework, effectiveness and cost-effectiveness and the weighting of benefits and harms. The Recommendations item focuses on how well the anticipated outcomes are described, the comprehensiveness of the guidance, ethical and equity considerations, and details for operationalising and updating the guidance. The final item, Implementability, is concerned with barriers to implementing the recommendations, resource and sustainability issues, how flexible/transferable the guidance is and the plan for disseminating, monitoring and evaluating the impact of the guidance. The AGREE-HS tool also includes two questions relating to overall assessment of the framework, specifically whether the reviewers would recommend the framework in the appropriate context and also their own context. A detailed description of the AGREE-HS items is included in Appendix 1.

Three researchers independently assessed each of the identified frameworks according to the instructions in the AGREE-HS user manual. 38 The researchers then met to reach a consensus score for each AGREE-HS item and the overall assessment of the frameworks. Final items scores were calculated using the summed consensus scores for each item. The highest score possible was 35 and the lowest score possible five.

[Refer to Appendix 1: Overview of AGREE-HS tool items]

\section{Data analysis}

AGREE-HS data were analysed descriptively. Total AGREE-HS scores for peer-reviewed and grey literature frameworks, and combined frameworks, presented as mean and standard deviation. Post-hoc analysis (independent samples t-test) was performed on the total and individual item scores for peer-reviewed and grey literature frameworks to enable comparison of the respective categories.

\section{Results}

The database search yielded a total of 1392 studies. Duplicates were removed and the remaining 1117 studies were screened by title and abstract. Six additional studies were included following a review of reference lists. After title and abstract screening, 84 studies were included for full text review. 


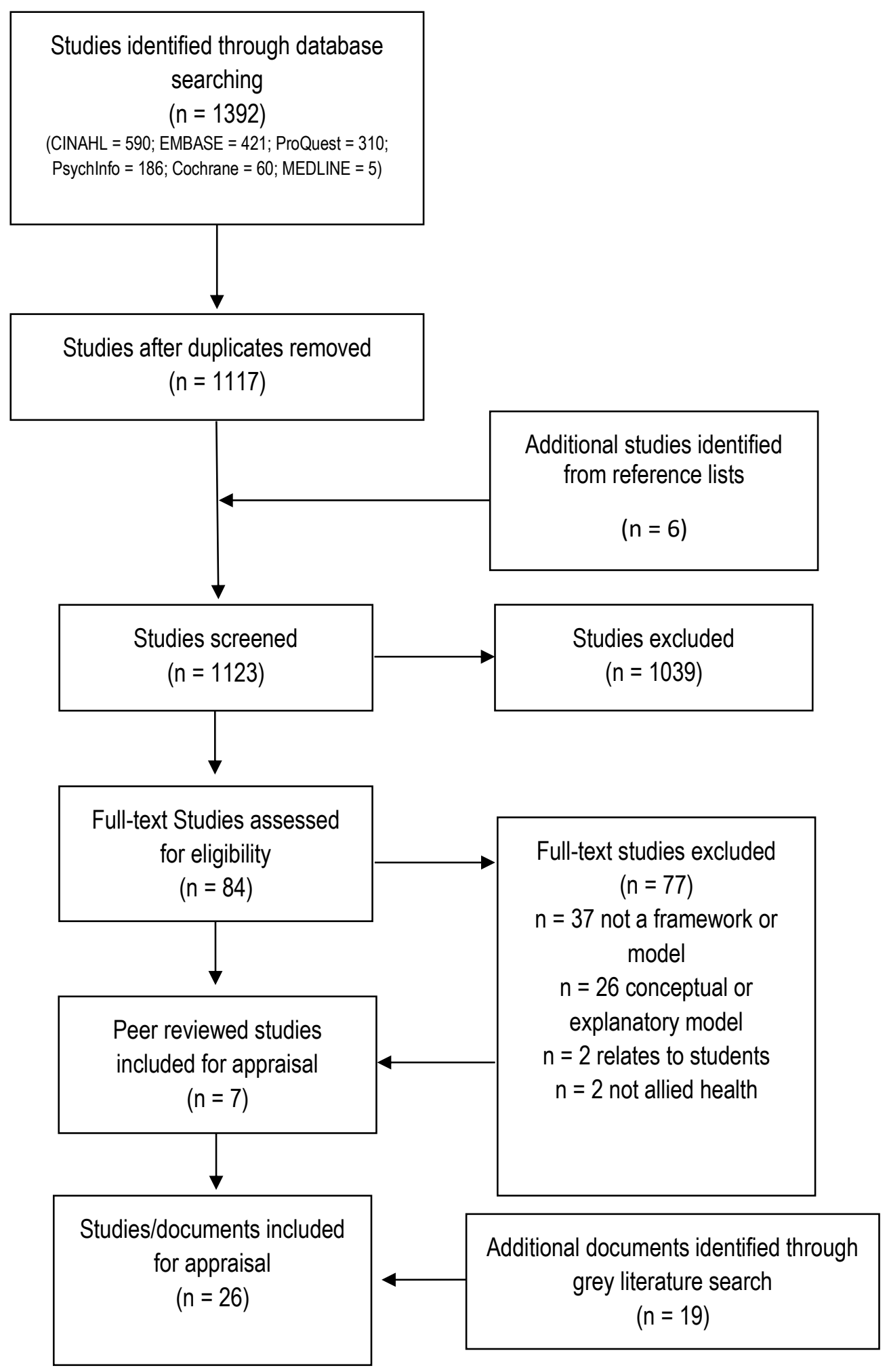

Figure 1: PRISMA flow chart 
Twenty-six studies describing conceptual models of clinical supervision relating to allied health were excluded. These models were mostly developed for psychology and social work. A total of 26 studies/documents were included for AGREE-HS appraisal, with seven peer-review studies and 19 grey literature documents.

The characteristics of the included peer-reviewed studies are described in Table 1. Three of the seven studies were from Australia. Five of the studies were broadly relevant for allied health working in the health and community services sectors. Three peer-reviewed studies were from social work, two were multidisciplinary while two were from psychology and occupational therapy.

Table 1: Characteristics of peer-reviewed studies included for AGREE-HS appraisal $(n=7)$

\begin{tabular}{|c|c|c|c|c|}
\hline Author/year & Title & Country & $\begin{array}{l}\text { Professions } \\
\text { included }\end{array}$ & Industry sector \\
\hline Lee et al., $2018^{42}$ & $\begin{array}{l}\text { Developing a Working } \\
\text { Model of Cross-Cultural } \\
\text { Supervision: A } \\
\text { Competence- and Alliance- } \\
\text { Based Framework }\end{array}$ & Canada & Social work & All sectors \\
\hline Dugmore et al., $2018{ }^{43}$ & $\begin{array}{l}\text { Systemic supervision in } \\
\text { statutory social work in the } \\
\text { UK: systemic rucksacks } \\
\text { and bells that ring }\end{array}$ & United Kingdom & Social work & Children's services \\
\hline O'Donoghue et al., 201844 & $\begin{array}{l}\text { Constructing an evidence- } \\
\text { informed social work } \\
\text { supervision model }\end{array}$ & $\begin{array}{l}\text { Unspecified } \\
\text { (authors from New } \\
\text { Zealand, Singapore } \\
\text { and Hong Kong) }\end{array}$ & Social work & All sectors \\
\hline Morris et al., 201745 & $\begin{array}{l}\text { A framework to support } \\
\text { experiential learning and } \\
\text { psychological flexibility in } \\
\text { supervision: SHAPE }\end{array}$ & Australia & Psychology & All sectors \\
\hline Nancarrow et al., 201430 & $\begin{array}{l}\text { Connecting practice: a } \\
\text { practitioner centred model } \\
\text { of supervision }\end{array}$ & Australia & Allied health & Rural health \\
\hline Brayman et al., 201446 & $\begin{array}{l}\text { Guidelines for Supervision, } \\
\text { Roles, and Responsibilities } \\
\text { During the Delivery of } \\
\text { Occupational Therapy } \\
\text { Services }\end{array}$ & United States & $\begin{array}{l}\text { Occupational } \\
\text { therapy }\end{array}$ & All sectors \\
\hline Hall et al., 201447 & $\begin{array}{l}\text { Professional support } \\
\text { framework: improving } \\
\text { access to professional } \\
\text { support for professionals }\end{array}$ & Australia & Allied health & Health \\
\hline
\end{tabular}

The characteristics of the included frameworks sourced from the grey literature are outlined in Table 2. Over half were from Australia and a third were from the United Kingdom. Five were developed for allied health, three specifically related to social work and psychology and two were specific for allied health professions working in mental health. The grey literature documents were mostly developed for a broad range of sectors, with seven relevant frameworks across health and community services and five for health care settings. The other main sectors represented were mental health and alcohol and other drugs services. Eight grey literature frameworks have been developed since 2014. 
Table 2: Characteristics of grey literature documents included for AGREE-HS appraisal $(n=19)$

\begin{tabular}{|c|c|c|c|c|c|}
\hline $\begin{array}{l}\text { Authorl } \\
\text { Organisation, Year }\end{array}$ & Title & Country & $\begin{array}{l}\text { Professions } \\
\text { included }\end{array}$ & Industry sector & $\begin{array}{l}\text { Commissioned/ } \\
\text { developed by }\end{array}$ \\
\hline $\begin{array}{l}\text { Occupational } \\
\text { Therapy Australia, } \\
201948\end{array}$ & $\begin{array}{l}\text { OT Australia } \\
\text { Professional } \\
\text { Supervision } \\
\text { Framework }\end{array}$ & Australia & $\begin{array}{l}\text { Occupational } \\
\text { therapy }\end{array}$ & All sectors & OT Australia \\
\hline $\begin{array}{l}\text { Victorian } \\
\text { Department of } \\
\text { Health and Human } \\
\text { Services, 2019 49 }\end{array}$ & $\begin{array}{l}\text { Victorian Allied Health } \\
\text { Clinical Supervision } \\
\text { Framework }\end{array}$ & Australia & $\begin{array}{l}\text { Allied health } \\
\text { (includes allied } \\
\text { health science } \\
\text { professions) }\end{array}$ & $\begin{array}{l}\text { Health and } \\
\text { community } \\
\text { services }\end{array}$ & DHHS Victoria \\
\hline $\begin{array}{l}\text { New South Wales } \\
\text { Health, } 2015^{33,50}\end{array}$ & $\begin{array}{l}\text { NSW Health Clinical } \\
\text { Supervision } \\
\text { Framework (including } \\
\text { The Superguide: a } \\
\text { handbook for } \\
\text { supervising allied } \\
\text { health professionals) }\end{array}$ & Australia & $\begin{array}{l}\text { Medicine, } \\
\text { nursing and } \\
\text { allied health }\end{array}$ & Health & $\begin{array}{l}\text { NSW Health - } \\
\text { Health Education } \\
\text { and Training } \\
\text { Institute }\end{array}$ \\
\hline $\begin{array}{l}\text { Roth et al., } 2015 \\
\text { [revised version] }\end{array}$ & $\begin{array}{l}\text { A competence } \\
\text { framework for the } \\
\text { supervision of } \\
\text { psychological } \\
\text { therapies }\end{array}$ & $\begin{array}{l}\text { United } \\
\text { Kingdom }\end{array}$ & Psychology & $\begin{array}{l}\text { Health and } \\
\text { community } \\
\text { settings }\end{array}$ & $\begin{array}{l}\text { Originally } \\
\text { commissioned for } \\
\text { NHS Education } \\
\text { for Scotland }\end{array}$ \\
\hline $\begin{array}{l}\text { Australian } \\
\text { Association of } \\
\text { Social Workers, } \\
201452\end{array}$ & $\begin{array}{l}\text { AASW Supervision } \\
\text { Standards }\end{array}$ & Australia & Social work & $\begin{array}{l}\text { Health and } \\
\text { community } \\
\text { settings }\end{array}$ & $\begin{array}{l}\text { Australian } \\
\text { Association of } \\
\text { Social Workers }\end{array}$ \\
\hline $\begin{array}{l}\text { South Australia } \\
\text { Health, } 201453\end{array}$ & $\begin{array}{l}\text { Allied Health Clinical } \\
\text { Supervision } \\
\text { Framework }\end{array}$ & Australia & $\begin{array}{l}\text { Allied health } \\
\text { professionals } \\
\text { (not further } \\
\text { specified) }\end{array}$ & Health & $\begin{array}{l}\text { SA Health Allied } \\
\text { and Scientific } \\
\text { Health Office }\end{array}$ \\
\hline $\begin{array}{l}\text { The British } \\
\text { Psychological } \\
\text { Society, } 2014{ }^{54}\end{array}$ & $\begin{array}{l}\text { DCP Policy of } \\
\text { Supervision }\end{array}$ & $\begin{array}{l}\text { United } \\
\text { Kingdom }\end{array}$ & $\begin{array}{l}\text { Clinical } \\
\text { psychologists }\end{array}$ & Health & $\begin{array}{l}\text { The British } \\
\text { Psychological } \\
\text { Society Division } \\
\text { of Clinical } \\
\text { Psychology }\end{array}$ \\
\hline $\begin{array}{l}\text { National } \\
\text { Association of } \\
\text { Social Workers and } \\
\text { The Association of } \\
\text { Social Work } \\
\text { Boards, } 201455\end{array}$ & $\begin{array}{l}\text { Best Practice } \\
\text { Standards in Social } \\
\text { Work Supervision }\end{array}$ & $\begin{array}{l}\text { United } \\
\text { Kingdom }\end{array}$ & Social work & $\begin{array}{l}\text { Health and } \\
\text { community } \\
\text { settings }\end{array}$ & $\begin{array}{l}\text { NASW and } \\
\text { ASWB }\end{array}$ \\
\hline
\end{tabular}




\begin{tabular}{|c|c|c|c|c|c|}
\hline $\begin{array}{l}\text { The British } \\
\text { Association of } \\
\text { Social Workers, } \\
2011^{56}\end{array}$ & UK Supervision Policy & $\begin{array}{l}\text { United } \\
\text { Kingdom }\end{array}$ & Social work & $\begin{array}{l}\text { Health and } \\
\text { community } \\
\text { settings }\end{array}$ & $\begin{array}{l}\text { The British } \\
\text { Association of } \\
\text { Social Workers }\end{array}$ \\
\hline $\begin{array}{l}\text { Government of } \\
\text { Western Australia } \\
\text { Drug and Alcohol } \\
\text { Office, } 2011{ }^{57}\end{array}$ & $\begin{array}{l}\text { Clinical Supervision } \\
\text { Handbook }\end{array}$ & Australia & Not specified & $\begin{array}{l}\text { Alcohol and other } \\
\text { drugs }\end{array}$ & $\begin{array}{l}\text { WA Drug and } \\
\text { Alcohol Office }\end{array}$ \\
\hline $\begin{array}{l}\text { New Zealand } \\
\text { Psychologists } \\
\text { Board, 2010 } 58\end{array}$ & $\begin{array}{l}\text { Guidelines on } \\
\text { Supervision }\end{array}$ & New Zealand & Psychology & $\begin{array}{l}\text { Health and } \\
\text { community } \\
\text { services }\end{array}$ & $\begin{array}{l}\text { New Zealand } \\
\text { Psychologists } \\
\text { Board }\end{array}$ \\
\hline $\begin{array}{l}\text { National Health } \\
\text { Service } \\
\text { Lanarkshire, } 201059\end{array}$ & $\begin{array}{l}\text { Professional/Clinical } \\
\text { Supervision Handbook } \\
\text { for Allied Health } \\
\text { Professionals }\end{array}$ & $\begin{array}{l}\text { United } \\
\text { Kingdom }\end{array}$ & $\begin{array}{l}\text { Allied Health } \\
\text { (Speech and } \\
\text { language } \\
\text { Therapy, } \\
\text { Occupational } \\
\text { Therapy, } \\
\text { Physiotherapy, } \\
\text { Audiology, } \\
\text { Dietetics, } \\
\text { Podiatry) }\end{array}$ & Healthcare & $\begin{array}{l}\text { National Health } \\
\text { Service } \\
\text { Lanarkshire }\end{array}$ \\
\hline $\begin{array}{l}\text { Queensland Health, } \\
200960\end{array}$ & $\begin{array}{l}\text { Clinical Supervision } \\
\text { Guidelines for Mental } \\
\text { Health Services }\end{array}$ & Australia & $\begin{array}{l}\text { Mental health } \\
\text { professions }\end{array}$ & Mental health & $\begin{array}{l}\text { Queensland } \\
\text { Health }\end{array}$ \\
\hline $\begin{array}{l}\text { Victorian } \\
\text { Healthcare } \\
\text { Association, } 200861\end{array}$ & $\begin{array}{l}\text { Clinical Supervision in } \\
\text { Community Health: } \\
\text { Introduction and } \\
\text { Practice Guidelines }\end{array}$ & Australia & Not specified & $\begin{array}{l}\text { Community } \\
\text { Health }\end{array}$ & $\begin{array}{l}\text { Victorian } \\
\text { Healthcare } \\
\text { Association } \\
\text { funded by the } \\
\text { Victorian } \\
\text { Department of } \\
\text { Human Services }\end{array}$ \\
\hline $\begin{array}{l}\text { Western Australia } \\
\text { Department of } \\
\text { Health [1], } 200862\end{array}$ & $\begin{array}{l}\text { Professional Support: } \\
\text { Clinical Supervision for } \\
\text { Allied Health } \\
\text { Professionals }\end{array}$ & Australia & $\begin{array}{l}\text { Allied health } \\
\text { professionals } \\
\text { (not further } \\
\text { specified) }\end{array}$ & Rural health & $\begin{array}{l}\text { WA Country } \\
\text { Health Service }\end{array}$ \\
\hline $\begin{array}{l}\text { College of Physical } \\
\text { Therapists of } \\
\text { Alberta, } 2008{ }^{63}\end{array}$ & $\begin{array}{l}\text { Supervision Resource } \\
\text { Guide for Physical } \\
\text { Therapists }\end{array}$ & Canada & $\begin{array}{l}\text { Physical } \\
\text { therapists }\end{array}$ & $\begin{array}{l}\text { Health and } \\
\text { community } \\
\text { services }\end{array}$ & $\begin{array}{l}\text { College of } \\
\text { Physical } \\
\text { Therapists of } \\
\text { Alberta }\end{array}$ \\
\hline
\end{tabular}




\begin{tabular}{|l|l|l|l|l|l|}
\hline Ask et al, 2008 64 & $\begin{array}{l}\text { Clinical Supervision: A } \\
\text { practical guide for the } \\
\text { alcohol and other } \\
\text { drugs field }\end{array}$ & Australia & $\begin{array}{l}\text { Alcohol and } \\
\text { other drugs } \\
\text { professions }\end{array}$ & $\begin{array}{l}\text { Alcohol and other } \\
\text { drugs }\end{array}$ & $\begin{array}{l}\text { National Center } \\
\text { for Training and } \\
\text { Education on } \\
\text { Addiction }\end{array}$ \\
\hline $\begin{array}{l}\text { Western Australia } \\
\text { Department of } \\
\text { Health [2], 2005 65 }\end{array}$ & $\begin{array}{l}\text { Clinical Supervision: } \\
\text { Framework for WA } \\
\text { Mental Health } \\
\text { Services and } \\
\text { Clinicians }\end{array}$ & Australia & $\begin{array}{l}\text { All clinical staff } \\
\text { in public } \\
\text { mental health } \\
\text { services }\end{array}$ & Mental health & $\begin{array}{l}\text { Western Australia } \\
\text { Department of } \\
\text { Health }\end{array}$ \\
\hline $\begin{array}{l}\text { Society of } \\
\text { Radiographers, } \\
\mathbf{2 0 0 3 6 6}\end{array}$ & $\begin{array}{l}\text { Radiography Clinical } \\
\text { Supervision } \\
\text { Framework }\end{array}$ & $\begin{array}{l}\text { United } \\
\text { Kingdom }\end{array}$ & Radiography & Healthcare & \\
\hline
\end{tabular}

A summary of the characteristics of all frameworks and comparisons of peer reviewed and grey literature frameworks are described in Table 3. The combined mean of the AGREE-HS final items scores for all studies/documents was $14.5(\mathrm{SD}=4.0)$ out of a possible total score of 35 . The mean of the final items scores was slightly higher for peer-reviewed studies ( $M=15.2$, $S D=3.5)$ than for grey literature documents $(M=14.3, S D=4.2)$. The mean scores of the AGREE-HS items (scored between 1 and 7) that were rated highest were for Topic $(M=3.3, S D=1.3)$ and Recommendations $(M=3.7, S D=1.3)$. When considered overall, the frameworks scored higher for items that involved providing rationale for the need for guidance in clinical supervision practice and having recommendations that could be operationalised. Lower scores were recorded for the AGREE-HS items relating to Participants $(M=2.5, S D=0.8)$, Methods $(M=2.6, S D=1.2)$ and Implementability $(M=2.7, S D=1.1)$. The items that were rated lower in quality overall were those relating to inadequate expertise and diversity amongst the developers, using appropriate evidence and systematic methods to inform the framework's development. The items relating to having strategies for implementation, such as those associated with cost and sustainability, and including methods for monitoring and evaluation, were rated lower across the combined peer-reviewed and grey literature frameworks.

Table 3: Summary of included studies and documents characteristics and comparison of means and standard deviations of AGREE-HS items $(n=26)$

\begin{tabular}{|c|c|c|c|c|c|c|c|c|}
\hline Professions included & Industry sector & Country & Topic & Participants & Methods & $\begin{array}{l}\text { Recommend- } \\
\text { ations }\end{array}$ & $\begin{array}{l}\text { Implement } \\
\text {-ability }\end{array}$ & Total \\
\hline \multicolumn{9}{|l|}{ Peer-reviewed $(n=7)$} \\
\hline $\begin{array}{l}\text { Social work = } 3 \\
\text { Allied health = } 2 \\
\text { Psychology = } 1 \\
\text { Occupational } \\
\text { therapy =1 }\end{array}$ & $\begin{array}{l}\text { All sectors = } 5 \\
\text { Children's } \\
\text { services }=1 \\
\text { Rural health = } 1\end{array}$ & $\begin{array}{l}\text { Australia = } 3 \\
\text { United } \\
\text { Kingdom }=1 \\
\text { Canada }=1 \\
\text { New } \\
\text { Zealand }=1 \\
\text { United } \\
\text { States }=1 \\
\text { International } \\
=1\end{array}$ & $\begin{array}{l}3.71 \\
(1.38)\end{array}$ & $\begin{array}{l}2.57 \\
(1.13)\end{array}$ & $\begin{array}{l}3.42 \\
(1.39)^{*}\end{array}$ & $\begin{array}{l}2.85 \\
(0.69)\end{array}$ & $\begin{array}{l}2.71 \\
(1.38)\end{array}$ & $\begin{array}{l}15.28 \\
(3.81)\end{array}$ \\
\hline \multicolumn{9}{|c|}{ Grey literature $(n=19)$} \\
\hline $\begin{array}{l}\text { Allied health }=5 \\
\text { Psychology }=3 \\
\text { Social work }=3\end{array}$ & $\begin{array}{l}\text { All sectors }=7 \\
\text { Health }=5\end{array}$ & $\begin{array}{l}\text { Australia = } \\
10\end{array}$ & $\begin{array}{l}3.15 \\
(1.25)\end{array}$ & $\begin{array}{l}2.42 \\
(0.76)\end{array}$ & $\begin{array}{l}2.31 \\
(1.00)\end{array}$ & $\begin{array}{l}3.68 \\
(1.20)^{*}\end{array}$ & $\begin{array}{l}2.68 \\
(1.05)\end{array}$ & $\begin{array}{l}14.26 \\
(4.27)\end{array}$ \\
\hline
\end{tabular}




\begin{tabular}{|c|c|c|c|c|c|c|c|c|}
\hline $\begin{array}{l}\text { Mental health = } 2 \\
\text { Medicine/nursing } \\
\text { and allied health = } 1 \\
\text { Occupational } \\
\text { therapy = } 1 \\
\text { Physiotherapy = } 1 \\
\text { Alcohol and other } \\
\text { drugs = } 1 \\
\text { Not specified = } 2\end{array}$ & $\begin{array}{l}\text { Mental health = } \\
2 \\
\text { Alcohol and } \\
\text { other drugs = } 2 \\
\text { Rural health = } 1 \\
\text { Community } \\
\text { health = } 1\end{array}$ & $\begin{array}{l}\text { United } \\
\text { Kingdom = } 6 \\
\text { Canada = } 1 \\
\text { New } \\
\text { Zealand = } 1\end{array}$ & & & & & & \\
\hline \multicolumn{9}{|c|}{ Combined frameworks $(n=26)$} \\
\hline $\begin{array}{l}\text { Allied health = } 7 \\
\text { Social work = } 6 \\
\text { Psychology = } 4 \\
\text { Occupational } \\
\text { therapy = } 2 \\
\text { Mental health = } 2 \\
\text { Medicine/nursing } \\
\text { and allied health = } 1 \\
\text { Alcohol and other } \\
\text { drugs = } 1 \\
\text { Not specified = } 2\end{array}$ & $\begin{array}{l}\text { All sectors = } 12 \\
\text { Health = } 5 \\
\text { Mental health = } \\
2 \\
\text { Rural health = } 2 \\
\text { Children's } \\
\text { services = } 1 \\
\text { Community } \\
\text { health = } 1\end{array}$ & $\begin{array}{l}\text { Australia = } \\
13 \\
\text { United } \\
\text { Kingdom = } 7 \\
\text { Canada = } 2 \\
\text { New } \\
\text { Zealand = } 2 \\
\text { United } \\
\text { States = 1 } \\
\text { International } \\
=1\end{array}$ & $\begin{array}{l}3.30 \\
(1.28)\end{array}$ & $\begin{array}{l}2.46 \\
(0.84)\end{array}$ & $\begin{array}{l}2.61 \\
(1.20)\end{array}$ & $\begin{array}{l}3.46 \\
(1.13)\end{array}$ & $\begin{array}{l}2.69 \\
(1.10)\end{array}$ & $\begin{array}{l}14.53 \\
(4.11)\end{array}$ \\
\hline
\end{tabular}

${ }^{*}$ Post-hoc analysis demonstrated difference in AGREE-HS scores between peer-reviewed and grey literature frameworks reached statistical significance $(p=0.05)$

The AGREE-HS scores for peer-reviewed and grey literature frameworks are shown in Table 4. When considering the peerreviewed studies alone, the highest individual items mean scores were for Topic $(M=3.7, S D=1.3)$ and Methods $(M=3.5, S D=$ 1.3), whereas for the grey literature documents, the highest individual items score was for Recommendations $(M=3.7, S D=1.2)$. Post-hoc analysis comparing the individual items mean scores for peer-reviewed and grey literature frameworks showed that there were significant differences between Methods and Recommendations. This indicates that the peer-reviewed studies scored higher in the item relating to using robust evidence and methods, such as systematic reviews, to inform the frameworks' recommendations. In contrast, the grey literature documents scored higher than the peer-reviewed studies in the item that relates to recommendations that were clear, succinct and easy to interpret. Individual items scores for Implementability and Participants were low for both peer-reviewed studies and grey literature documents.

Table 4: AGREE-HS appraisal of peer-reviewed studies $(n=7)$ and grey literature documents $(n=19)$

\begin{tabular}{|c|c|c|c|c|c|c|}
\hline Author/year & Topic & Participants & Methods & Recommendations & Implementability & Total score \\
\hline \multicolumn{7}{|l|}{ Peer-reviewed } \\
\hline Lee et al., 2018 & 4 & 1 & 4 & 4 & 3 & 16 \\
\hline Dugmore et al., 2018 & 5 & 3 & 3 & 3 & 4 & 18 \\
\hline O'Donoghue et al., 2018 & 3 & 2 & 5 & 3 & 2 & 15 \\
\hline Morris et al., 2017 & 3 & 2 & 3 & 3 & 2 & 13 \\
\hline Nancarrow et al., 2014 & 6 & 4 & 5 & 2 & 2 & 19 \\
\hline Brayman et al., 2014 & 2 & 2 & 1 & 2 & 1 & 8 \\
\hline Hall et al., 2013 & 3 & 4 & 3 & 3 & 5 & 18 \\
\hline \multicolumn{7}{|l|}{ Grey literature } \\
\hline $\begin{array}{l}\text { Occupational Therapy } \\
\text { Australia, } 2019\end{array}$ & 6 & 2 & 5 & 5 & 5 & 23 \\
\hline $\begin{array}{l}\text { Victorian Department of Health } \\
\text { and Human Services, } 2019\end{array}$ & 4 & 3 & 4 & 5 & 4 & 20 \\
\hline
\end{tabular}




\begin{tabular}{|c|c|c|c|c|c|c|}
\hline NSW Health, 2015 & 4 & 4 & 2 & 5 & 3 & 18 \\
\hline Roth et al., 2015 & 4 & 3 & 4 & 4 & 4 & 19 \\
\hline $\begin{array}{l}\text { Australian Association of } \\
\text { Social Workers, } 2014\end{array}$ & 2 & 2 & 2 & 5 & 2 & 13 \\
\hline SA Health, 2014 & 3 & 2 & 2 & 4 & 4 & 15 \\
\hline $\begin{array}{l}\text { The British Psychological } \\
\text { Society, } 2014\end{array}$ & 3 & 3 & 2 & 5 & 3 & 16 \\
\hline $\begin{array}{l}\text { National Association of Social } \\
\text { Workers and The Association } \\
\text { of Social Work Boards, } 2013\end{array}$ & 2 & 3 & 2 & 4 & 2 & 13 \\
\hline $\begin{array}{l}\text { The British Association of } \\
\text { Social Workers, } 2011\end{array}$ & 4 & 2 & 2 & 3 & 2 & 13 \\
\hline $\begin{array}{l}\text { Government of Western } \\
\text { Australia Drug and Alcohol } \\
\text { Office, } 2011\end{array}$ & 1 & 1 & 2 & 2 & 1 & 7 \\
\hline $\begin{array}{l}\text { New Zealand Psychologists } \\
\text { Board, } 2010\end{array}$ & 2 & 2 & 1 & 2 & 2 & 9 \\
\hline NHS Lanarkshire, 2010 & 4 & 4 & 3 & 4 & 3 & 18 \\
\hline Queensland Health, 2009 & 4 & 2 & 2 & 4 & 3 & 15 \\
\hline $\begin{array}{l}\text { Victorian Healthcare } \\
\text { Association, } 2008\end{array}$ & 3 & 2 & 2 & 3 & 2 & 12 \\
\hline $\begin{array}{l}\text { College of Physical Therapists } \\
\text { of Alberta, } 2008\end{array}$ & 2 & 2 & 1 & 2 & 1 & 8 \\
\hline $\begin{array}{l}\text { Western Australia Department } \\
\text { of Health [1], } 2008\end{array}$ & 3 & 2 & 2 & 2 & 2 & 11 \\
\hline Ask et al., 2005 & 5 & 3 & 2 & 4 & 3 & 17 \\
\hline $\begin{array}{l}\text { Western Australia Department } \\
\text { of Health [2], } 2003\end{array}$ & 2 & 2 & 2 & 5 & 3 & 14 \\
\hline Society of Radiographers, 2003 & 1 & 1 & 2 & 2 & 1 & 7 \\
\hline
\end{tabular}

The frameworks which received the highest scores were developed by Occupational Therapy Australia ${ }^{23}$ and the Victorian Department of Health and Human Services ${ }^{20}$. These frameworks were both grey literature documents from Australia. The highest scores for frameworks from peer-reviewed studies were those developed by Nancarrow et al. ${ }^{30}$ and Hall et al. ${ }^{47}$, which were also Australian.

\section{Discussion}

Clinical supervision frameworks for allied health are mostly located in the grey literature. The models and frameworks described in peer-reviewed literature were more conceptual or theoretical. Most frameworks included in this review were multidisciplinary and where specified, were likely to have been developed for allied health professions including physiotherapy, occupational therapy, social work, dietetics, speech pathology, psychology and podiatry. Where frameworks were developed for an individual profession, these were mostly for social work or psychology. The frameworks often lacked detail describing the sectors that they were developed for, but they were often broadly relevant for allied health professions working in health and community services sectors. Specific sectors included mental health and alcohol and other drug services.

The majority of frameworks appraised were developed in Australia, which is consistent with much of the published clinical supervision research for allied health professions in the last decade.., 67 The need for practical allied health clinical supervision frameworks to improve the quality of clinical supervision practice has been a recommendation from a number of recent Australian studies. ${ }^{26,37,68,69}$ This may partly explain the number of frameworks developed by Australian state governments and professional bodies since 2014.

The quality of the frameworks reviewed was variable and generally low. There was little difference between the overall quality of peer-review studies and those from the grey literature aside from the individual items relating to Methods and Recommendations. As expected, peer-reviewed studies used more robust evidence and methods to inform their development, including systemic reviews. Many grey literature documents included little or no description of how evidence informed their development and used limited referencing of research evidence. Recommendations made in grey literature documents were often clearly outlined, 
succinct, practical and flexible for stakeholders to implement. When considering the development of future clinical supervision frameworks, a combination of the respective strengths of peer-reviewed studies (more robust use of evidence to inform the framework development) and grey literature documents (providing clear and practical recommendations) would result in better overall quality.

The application of the AGREE-HS items highlighted common methodological flaws across the frameworks. Most frameworks did not factor in cost of supervision and considerations around the cost-effectiveness of framework implementation. The costs of clinical supervision are often difficult to calculate due to being unable to separate time spent in clinical supervision from clinical practice and a lack of tangible outcomes from clinical supervision. $9,70,71 \mathrm{~A}$ better estimate of costs is needed in relation to benefits that may assist stakeholders, such as health service organisations, to prioritise the implementation of framework recommendations for allied health.

Few frameworks referenced principles relating to professional ethics and diversity in their development or recommendations. Therefore, factors relating to gender, race, culture and vulnerable groups of workers and their patients may not be adequately addressed in clinical supervision. ${ }^{72-74}$ Many frameworks did not consider factors that would enhance their implementation and sustainability. These included the anticipated barriers of introducing the framework, methods and tools for evaluating its impact and plans for updating the framework. This may explain the reported poor uptake of clinical supervision frameworks for allied health. 7,19

Relatively simple considerations could improve the quality of clinical supervision frameworks for allied health. Many frameworks did not describe who developed the framework, however, when these were described, there was often a lack of diversity of the developers/contributors, including lack of multidisciplinary or participants from various sectors and lack of service consumer input. Ensuring that a range of stakeholders have input into the development of frameworks and adequate description of their roles and affiliations, would improve the credibility and trustworthiness. ${ }^{75}$ Many frameworks did not adequately describe how consensus was reached in the development of recommendations. Use of consensus methods by framework developers such as Delphi or stakeholder reference groups, could ensure that recommendations are relevant and acceptable for their audience, and are able to be practically implemented. ${ }^{76}$

The developers of the AGREE-HS tool specify that the tool can be used to assist in the development of new frameworks. ${ }^{77}$ Using such a tool to guide framework development could improve framework quality, by drawing attention to identified weaknesses, such as representative selection of developers and use of robust and transparent methods. Another potential advantage of using such a tool would be to improve evaluation methods of framework implementation. Evaluation was rarely considered across frameworks and needs to be included in the future for continuous quality improvement and successful implementation.

There are now many local jurisdictions, such as government agencies or professional bodies, who have developed clinical supervision frameworks for allied health. However, there are still significant gaps in framework availability, depending on sector and profession, and the frameworks that do exist are of variable quality. There are several potential reasons for the framework limitations including lack of visibility of clinical supervision and reduced recognition of allied health on the health policy agenda, ${ }^{1,77}$ flawed processes for developing health policy, ${ }^{78}$ limited resources to assist in framework development and unclear evidence to support positive patient outcomes from clinical supervision. 8,9 As recommended by Fitzpatrick, a pragmatic way to address these barriers would be to develop national allied health clinical supervision frameworks which apply across professions and jurisdictions. ${ }^{19}$ This policy initiative would require robust and inclusive methods for development and proper consideration of implementation and evaluation. ${ }^{78}$ Such frameworks could be broad, flexible and overarching, complemented by guidelines for individual professions that outline any profession-specific approaches to clinical supervision. Of the existing frameworks, those from Occupational Therapy Australia and Victorian Department of Health and Human Services provide examples of a professionspecific and an interprofessional framework as a basis for the development of a common allied health clinical supervision framework.

The recently developed AGREE-HS tool has had limited application thus far, having been used to assess the quality of health system guidance documents such as those from the World Health Organisation and the National Institute for Clinical Excellence..$^{79}$ This is the first time that the authors are aware that the tool has been used for a specific health system topic such as clinical supervision, and the first time that the AGREE-HS appraisal has included peer-reviewed studies and frameworks developed for specific organisations or professional associations. The authors perceived that the AGREE-HS tool was easy and relatively time efficient to use with the item descriptions flexible enough to be relevant and applicable for frameworks described in peer-reviewed studies and grey literature documents. Initially there was variation in the individual reviewer's scores for particular items. The reviewer discussions of scores to reach consensus assisted standardisation of the approach to scoring, reducing variation. The reviewers recommend this consensus method for those using the AGREE-HS tool in the future. While the 
reviewers included the overall assessment questions relating to whether they would recommend the framework in their context/the appropriate context for their appraisal, these questions would be more useful to inform agencies on whether they would use a specific framework rather than as an objective assessment of the frameworks' quality. The developers of the AGREE-HS stipulate that the elements within each item can be ranked or modified a priori. This has been suggested as a mechanism to strengthen other appraisal tools and, although not undertaken for this review, would help to prioritise which aspects of frameworks have a stronger influence on the overall assessment of quality. ${ }^{80}$

There were limitations associated with this review. While authors tried to locate difficult to source frameworks through citation tracking, searching of government and professional association websites, and hand searching, existing frameworks may have been omitted, particularly those in the grey literature. Frameworks were appraised using only the information available within the document, with supplementary information included only if referred to in the document and where this information was publicly available. Some of the frameworks may have included this information elsewhere, such as details regarding the authors' credentials or methods of development, which was not used in the appraisal process. Two of the reviewers had potential conflicts of interest regarding the frameworks appraised. One reviewer was project manager for the development of one the frameworks, and another reviewer is a current president of a healthcare professional association. While the reviewer did not appraise the frameworks where there was a conflict of interest, this may have been a potential source of bias.

\section{Conclusion}

There are limited published frameworks available for allied health professionals and the frameworks that do exist are generally of low quality and are poorly evaluated. As a result, many existing frameworks may not provide the practical guidance required to improve clinical supervision practice and optimize the benefits of clinical supervision, such as improved clinical governance and health professional wellbeing. It is recommended that future policies relating to clinical supervision need to focus on the development of common, evidence-based allied health clinical supervision frameworks. Future frameworks should be practically orientated and need to use robust methods and evaluation to inform their development and implementation.

\section{References}

1. Philip K. Allied health: untapped potential in the Australian health system. Australian Health Review. 2015;39(3):244-7.

2. Davis SF, Enderby P, Harrop D, Hindle L. Mapping the contribution of Allied Health Professions to the wider public health workforce: a rapid review of evidence-based interventions. Journal of Public Health. 2017;39(1):177-83.

3. Nancarrow SA, Young G, O'Callaghan K, Jenkins M, Philip K, Barlow K. Shape of allied health: an environmental scan of 27 allied health professions in Victoria. Australian Health Review. 2017;41(3):327-35.

4. National Health Service. Health Education England Allied Health Professions. 2020. Available from: https://www.hee.nhs.uk/our-work/allied-health-professions.

5. Association of Schools Advancing Allied Health Professions. What is Allied Health? 2020. Available from: http://www.asahp.org/what-is/.

6. Dawson M, Phillips B, Leggat S. Clinical supervision for allied health professionals: a systematic review. Journal of allied health. 2013;42(2):65-73.

7. Milne D. An empirical definition of clinical supervision. Br J Clin Psychol. 2007;46(Pt 4):437-47. PubMed PMID: 17535535.

8. Snowdon D, Hau R, Leggat SG, Taylor NF. Does clinical supervision of health professionals improve patient safety? A systematic review and meta-analysis. International Journal for Quality in Health Care. 2016. doi: 10.1093/intghc/mzw059.

9. Snowdon DA, Leggat SG, Taylor NF. Does clinical supervision of healthcare professionals improve effectiveness of care and patient experience? A systematic review. BMC health services research. 2017;17(1):786.

10. White E, Winstanley J. Clinical supervision and the helping professions: An interpretation of history. The Clinical Supervisor. 2014;33(1):3-25.

11. Duckett S. Targeting zero: Supporting the Victorian hosptial system to eliminate avoidable harm and strengthen quality of care. Review of Hospital Safety and Quality Assurance in Victoria. 2016.

12. Henderson J, Willis E. Chapter Twelve: The Marketisation of Aged Care: The Impact of Aged Care Reform in Australia. Navigating private and public healthcare: Springer; 2020. p. 249-67.

13. Gilchrist DJ, Knight P, Edmonds C, Emery T. Six Years and Counting: The NDIS and the Australian Disability Services System-A White Paper. Available at SSRN 3571104. 2020.

14. Leggat SG, Gough R, Bartram T, Stanton P, Bamber GJ, Ballardie R, et al. Process redesign for time-based emergency admission targets. Journal of health organization and management. 2016. 
15. Andrikopoulos S, Johnson G. The Australian response to the COVID-19 pandemic and diabetes-lessons learned. Diabetes Research and Clinical Practice. 2020:108246.

16. Duckett S. What should primary care look like after the COVID-19 pandemic? Australian Journal of Primary Health. 2020.

17. Wells J. The impact of stress amongst health professionals. Taylor \& Francis; 2011.

18. Kadushin A, Harkness D. Supervision in social work: Columbia University Press; 2014.

19. Fitzpatrick S, Smith M, Wilding C. Quality allied health clinical supervision policy in Australia: a literature review. Australian Health Review. 2012;36(4):4-465 2p. doi: 10.1071/AH11053. PubMed PMID: 104445337.

20. Pearce $P$, Phillips $B$, Dawson $M$, Leggat $S$. Content of clinical supervision sessions for nurses and allied health professionalsA systematic review. Clinical Governance: An International Journal. 2013;18(2):139-54 16p. doi: 10.1108/14777271311317927. PubMed PMID: 104277411.

21. Pearce P, Phillips B, Dawson M, Leggat SG. Content of clinical supervision sessions for nurses and allied health professionals. Clinical Governance: An International Journal. 2013.

22. 2011;9(48 Suppl):52-65.

23. O'Toole $\mathrm{K}$, Schoo A, Hernan A. Why did they leave and what can they tell us? Allied health professionals leaving rural settings. Australian health review. 2010;34(1):66-72.

24. Snowdon D, Millard G, Taylor NF. Effectiveness of Clinical Supervision of Allied Health Professionals. J Allied Health. 2016;45(2):113-21. PubMed PMID: 116154769.

25. Dawson M, Phillips B, Leggat S. Effective clinical supervision for regional allied health professionals - the supervisee's perspective. Australian Health Review. 2012;36(1):92-7 6p. doi: 10.1071/AH11006. PubMed PMID: 104527820.

26. Gardner MJ, McKinstry C, Perrin B. Effectiveness of allied health clinical supervision a cross-sectional survey of supervisees. Journal of allied health. 2018;47(2):126-32.

27. Saxby C, Wilson J, Newcombe P. Can clinical supervision sustain our workforce in the current healthcare landscape? Findings from a Queensland study of allied health professionals. Australian Health Review. 2015;39(4):476-82. doi: http://dx.doi.org/10.1071/AH14183. PubMed PMID: 1776260253.

28. Milne D. Evidence-based clinical supervision. Chichester: BPS Blackwell. 2009.

29. Leggat S, Phillips B, Pearce P, Dawson M, Schulz D, Smith J. Clinical supervision for allied health staff: necessary but not sufficient. Australian Health Review. 2016;40(4):431-7. doi: 10.1071/AH15080. PubMed PMID: 117242443.

30. A. Nancarrow S, Wade R, Moran A, Coyle J, Young J, Boxall D. Connecting practice: a practitioner centred model of supervision. Clinical Governance: An International Journal. 2014;19(3):235-52.

31. Fitzpatrick S, Smith M, Wilding C. Clinical Supervision in Allied Health in Australia: A Model of Allied Health Clinical Supervision Based On Practitioner Experience. Internet Journal of Allied Health Sciences \& Practice. 2015;13(4):1-12 4p. PubMed PMID: 111917970.

32. Health Workforce Australia. National Clinical Supervision Support Framework. 2011. Available at: http://www.google.com/url?sa=t\&rct=i\&q=\&esrc=s\&source=web\&cd=9\&cad=rja\&uact=8\&ved=2ahUKEwi8n7Mw4DgAhWaXisKHTcOD3UQFjAlegQIAhAC\&url=http\%3A\%2F\%2Fintranet.heti.nsw.gov.au\%2FGlobal\%2Falliedhealth\%2FNSW\%2520Health\%2520Clinical\%2520Supervision\%2520Framework.pdf\&usg=AOvVaw2ljPDzTrMvSZzZv k6Q_pAy

33. New South Wales Health. NSW Health Clinical Supervision Framework. 2015. Available at: http://www.google.com/url?sa=t\&rct=i\&q=\&esrc=s\&source=web\&cd=9\&cad=rja\&uact=8\&ved=2ahUKEwi8n7Mw4DgAhWaXisKHTcOD3UQFjAlegQIAhAC\&url=http\%3A\%2F\%2Fintranet.heti.nsw.gov.au\%2FGlobal\%2Falliedhealth\%2FNSW\%2520Health\%2520Clinical\%2520Supervision\%2520Framework.pdf\&usg=AOvVaw2ljPDzTrMvSZzZv k6Q pAy

34. $\quad$ SA Health. Allied Health Clinical Supervision Framework. 2014. Available at: https://www.heti.nsw.gov.au/_data/assets/pdf_file/0005/424859/Superguide-May-2012.pdf

35. $\quad$ Nilsen P. Making sense of implementation theories, models and frameworks. Implementation science. 2015;10(1):53.

36. Lanarkshire NHS. Professional/Clinical Supervision Handbook for Allied Health Professionals. 2010.

37. Slade SC, Philip K, Morris ME. Frameworks for embedding a research culture in allied health practice: a rapid review. Health research policy and systems. 2018;16(1):29.

38. AGREE-HR Team. The Appraisal of Guidelines Research \& Evaluation-Health Systems (AGREE-HS) [Electronic version]. 2018. Sourced from: https://www.agreetrust.org/resource-centre/agree-hs/

39. Moher D, Liberati A, Tetzlaff J, Altman DG, Prisma Group. Preferred reporting items for systematic reviews and metaanalyses: the PRISMA statement. PLoS med. 2009 Jul 21;6(7).

40. Bhatt M, Nahari A, Wang P-W, Kearsley E, Falzone N, Chen S, et al. The quality of clinical practice guidelines for management of pediatric type 2 diabetes mellitus: a systematic review using the AGREE II instrument. Systematic reviews. 2018;7(1):193. 
41. Brouwers MC, Kho ME, Browman GP, Burgers JS, Cluzeau F, Feder G, et al. Development of the AGREE II, part 2: assessment of validity of items and tools to support application. Canadian Medical Association Journal. 2010;182(10):E472-E8.

42. Lee E, Kealy D. Developing a Working Model of Cross-Cultural Supervision: A Competence- and Alliance-Based Framework. Clinical Social Work Journal. 2018;46(4):310-20. doi: http://dx.doi.org/10.1007/s10615-018-0683-4. PubMed PMID: 2110393198.

43. Dugmore P, Partridge K, Sethi I, Krupa-Flasinska M. Systemic supervision in statutory social work in the UK: systemic rucksacks and bells that ring. European Journal of Social Work. 2018;21(3):400-14. doi: 10.1080/13691457.2018.1446914. PubMed PMID: 128681968.

44. O'Donoghue K, Wong Yuh Ju P, Tsui M-s. Constructing an evidence-informed social work supervision model. European Journal of Social Work. 2018;21(3):348-58. doi: 10.1080/13691457.2017.1341387. PubMed PMID: 128681960.

45. Morris EM, Bilich-Eric L. A framework to support experiential learning and psychological flexibility in supervision: SHAPE. Australian Psychologist. 2017;52(2):104-13. doi: http://dx.doi.org/10.1111/ap.12267. PubMed PMID: 201712996-003.

46. Brayman SJPOTRLF, Clark GFMSOTRLF, DeLany JVDOTRL, Garza ERPOTRATP, Radomski MVMAOTRLF, Ramsey RMSOTRL, et al. Guidelines for Supervision, Roles, and Responsibilities During the Delivery of Occupational Therapy Services. The American Journal of Occupational Therapy. 2014;68:S16-S22. doi: http://dx.doi.org/10.5014/ajot.2014.686S03. PubMed PMID: 1635433348.

47. Hall FBGD, Bell KBGG. Professional support framework: improving access to professional support for professionals. Australian Health Review. 2013;37(5):560-5. PubMed PMID: 1556323001; 23680624.

48. Occupational Therapy Australia. Professional Supervision Framework. 2019. Available at: https://otaus.com.au/publicassets/2e35a9f6-b890-e911-a2c39b7af2531dd2/ProfessionalSupervisionFramework2019.pdf

49. Department of Health and Human Services Victoria DoHaHS. Victorian allied health clinical supervision framework. 2019. Available at: https://www2.health.vic.gov.au/health-workforce/allied-health-workforce/clinical-supervisionframework

50. Health Education and Training Unit. The Superguide: A handbook for supervisiong allied health professionals. 2012. New South Wales Health. Available at: https://www.heti.nsw.gov.au/_data/assets/pdf_file/0005/424859/HETI_Superguide_Txt_WARAHETI_OCT_19.pdf

51. Roth $A D$ and Pilling S. A competence framework for the supervision of psychological therapies. 2015. Available at: https://www.ucl.ac.uk/pals/research/clinical-educational-and-health-psychology/research-groups/core/competenceframeworks

52. Australian Association of Social Workers. Supervision Standards. 2014. Available at: https://www.aasw.asn.au/document/item/6027

53. South Australia Health. Allied Health Clinical Supervision Framework. 2014. Available at: https://www.sahealth.sa.gov.au/wps/wcm/connect/public+content/sa+health+internet/clinical+resources/allied+and+sci entific+health/professional+development+and+training+in+allied+health\#SA Health Allied Health Clinical Supervision Framework

54. The British Psychological Society. DCP Policy of Supervision. 2014. Available at: https://www.google.com/url?sa=t\&rct=j\&q=\&esrc=s\&source=web\&cd=15\&cad=rja\&uact=8\&ved=2ahUKEwilj5C9wIDgA hWPWXOKHSujABI4ChAWMAR6BAgAEAI\&url=https $\% 3$ A $\% 2 F \% 2$ Fwww1.bps.org.uk $\% 2$ Fsystem $\% 2 F$ files $\% 2 F P u b l i c \%$ 2520files\%2Finf224_dcp_supervision.pdf\&usg=AOvVaw2XCYNmCQS7jciptV9F70cF

55. National Association of Social Workers and The Association of Social Work Boards. Best Practice Standards in Social Work Supervision. 2013. Available at:

https://www.socialworkers.org/LinkClick.aspx?fileticket=GBrLb|4Buwl\%3D\&portalid=0

56. The British Association of Social Workers. UK Supervision Policy. 2011. Available at: https://www.basw.co.uk/resources/basw-uk-supervision-policy

57. Government of Western Australia Drug and Alcohol Office. Clinical Supervision Handbook. 2011. Available at: https://www.google.com/url?sa=t\&rct=i\&q=\&esrc=s\&source=web\&cd=1\&cad=rja\&uact=8\&ved=2ahUKEwijvL_CxYDgA hWYV30KHX62AtAQFiAAegQIChAC\&url=https\%3A\%2F\%2Fwww.mhc.wa.gov.au\%2Fmedia\%2F1172\%2Ffinalclinical-supervision-handbook-8-jan-2013.pdf\&usg=AOvVaw2NF4w6qAfHYleCzWMBYggg

58. New Zealand Psychologists Board. Guidelines on Supervision. 2010. Available at: http://www.google.com/url?sa=t\&rct=i\&q=\&esrc=s\&source=web\&cd=14\&cad=ria\&uact=8\&ved=2ahUKEwi3hcDFvoDgA hVFWysKHQgzCHAQFjANegQIARAC\&url=http\%3A\%2F\%2Fwww.psychologistsboard.org.nz\%2Fcms_show_downloa d.php\%3Fid\%3D220\&usg=AOvVaw3ls3zQlbVsHeJs qUAvQoA 
59. National Health Service Lanarkshire. Professional/Clinical Supervision Handbook for Allied Health Professionals. 2010. Available at:

http://www.google.com/url?sa=t\&rct=i\&q=\&esrc=s\&source=web\&cd=1\&cad=rja\&uact=8\&ved=2ahUKEwiJq5mVxYDgA hUXf30KHXweBfQQFiAAegQIChAC\&url=http\%3A\%2F\%2Fwww.careerframework.nes.scot.nhs.uk\%2Fmedia\%2F5052 3\%2Fprof-clini-super-_ahps _2 nhsl_2010.pdf\&usg=AOvVaw0k3D AFsxk0oeERpV5IAmQ

60. Queensland Health. Clinical Supervision Guidelines for Mental Health Services. 2009. Available at: https://www.health.gld.gov.aul data/assets/pdf file/0026/371627/superguide_2009.pdf

61. Victorian Healthcare Association. Clinical Supervision in Community Health: Introduction and Practice Guidelines. 2008. Available at:

http://www.google.com/url?sa=t\&rct=i\&q=\&esrc=s\&source=web\&cd=1\&ved=2ahUKEwiGuM6mxoDgAhVDJHIKHdc6A bAQFjAAegQIARAC\&url=http\%3A\%2F\%2Fhealthcaregovernance.org.au\%2Fdocs\%2Fclinical-supervision--guidelines28final-29.doc\&usg=AOvVaw3w4Ro3Xj6xoPIGHVAQCDAf

62. Western Australia Department of Health. Professional Support: Clinical Supervision for Allied Health Professionals. 2008. Available at:

http://www.wacountry.health.wa.gov.au/fileadmin/sections/allied_health/WACHS_R_AH_ProSupport_ClinSupervision.p $\underline{\mathrm{df}}$

63. College of Physical Therapists of Alberta. Supervision Resource Guide for Physical Therapists. 2008. Available at: http://www.google.com/url?sa=t\&rct=i\&q=\&esrc=s\&source=web\&cd=40\&cad=rja\&uact=8\&ved=2ahUKEwiLg8XjwIDgA hUlfSsKHcQhCt84HhAWMAl6BAgBEAl\&url=http\%3A\%2F\%2Fphysicaltherapy.med.ubc.ca\%2Ffiles\%2F2012\%2F05\% 2FAlberta-College-Supervision-Resource-Guide.pdf\&usg=AOvVaw2nycqz6aGsFXib4NViaEj2

64. Ask A. and Roche M. Clinical Supervision: A practical guide for the alcohol and other drugs field. 2005. Available at: http://nceta.flinders.edu.au/files/7312/5728/7200/TheGuide.pdf

65. Western Australia Department of Health. Clinical Supervision: Framework for WA Mental Health Services and Clinicians. 2005. Available at: https://www.google.com/url?sa=t\&rct=i\&q=\&esrc=s\&source=web\&cd=76\&cad=rja\&uact=8\&ved=2ahUKEwirn_ypvoDg AhWDfysKHRbkBao4RhAWMAV6BAgIEAl\&url=https\%3A\%2F\%2Fwww.mhc.wa.gov.au\%2Fmedia\%2F1172\%2Ffinalclinical-supervision-handbook-8-jan-2013.pdf\&usg=AOvVaw2NF4w6qAfHYleCzWMBYggg

66. Society of Radiographers. Radiography Clinical Supervision Framework. 2003. Available at: https://www.sor.org/system/files/article/201202/sor_clinical_supervision_framework.pdf

67. Ducat WH, Kumar S. A systematic review of professional supervision experiences and effects for allied health practitioners working in non-metropolitan health care settings. Journal of multidisciplinary healthcare. 2015;8:397.

68. Snowdon DA, Millard G, Taylor NF. Effectiveness of clinical supervision of allied health professionals: a survey. Journal of allied health. 2016;45(2):113-21.

69. Saxby C, Wilson J, Newcombe P. Does best practice clinical supervision lead to better outcomes? Findings from a Queensland study of community allied health professionals. Advances in Clinical Supervision Innovation \& Practice. 2013:23.

70. White E, Winstanley J. Cost and resource implications of clinical supervision in nursing: an Australian perspective. Journal of Nursing Management. 2006;14(8):628-36.

71. White E, Winstanley J. A randomised controlled trial of clinical supervision: Selected findings from a novel Australian attempt to establish the evidence base for causal relationships with quality of care and patient outcomes, as an informed contribution to mental health nursing practice development. Journal of Research in Nursing. 2010;15(2):15167.

72. Arczynski AV, Morrow SL. The complexities of power in feminist multicultural psychotherapy supervision. Journal of Counseling Psychology. 2017;64(2):192-205. doi: http://dx.doi.org/10.1037/cou0000179. PubMed PMID: 2016-58885001.

73. Constantine MG, Warren AK, Miville ML. White racial identity dyadic interactions in supervision: Implications for supervisees' multicultural counseling competence. Journal of Counseling Psychology. 2005;52(4):490-6. doi: http://dx.doi.org/10.1037/0022-0167.52.4.490. PubMed PMID: 41587091.

74. Moreno JR. Multicultural events within group supervision: Minority experiences and supervision satisfaction. Dissertation Abstracts International Section A: Humanities and Social Sciences. 2010;70(7-A):2399. PubMed PMID: 2010-99010-430.

75. Beddoe L, Karvinen-Niinikoski S, Ruch G, Tsui M-s. Towards an international consensus on a research agenda for social work supervision: Report on the first survey of a Delphi study. The British journal of social work. 2016;46(6):1568-86.

76. Bourrée F, Michel P, Salmi LR. Consensus methods: review of original methods and their main alternatives used in public health. Revue d'epidemiologie et de sante publique. 2008;56(6):e13-e21. 
77. White E. Clinical Supervision: invisibility on the contemporary nursing and midwifery policy agenda. J Adv Nurs. 2017;73(6):1251-4.

78. White E. Claims to the benefits of clinical supervision: A critique of the policy development process and outcomes in New South Wales, Australia. Int J Ment Health Nurs. 2017;26(1):65-76.

79. Brouwers MC, Lavis JN, Spithoff K, Vukmirovic M, Florez ID, Velez M, et al. Assessment of health systems guidance using the Appraisal of Guidelines for Research and Evaluation-Health Systems (AGREE-HS) instrument. Health Policy. 2019;123(7):646-51.

80. Hoffmann-Eßer W, Siering U, Neugebauer EA, Brockhaus AC, McGauran N, Eikermann M. Guideline appraisal with AGREE II: online survey of the potential influence of AGREE II items on overall assessment of guideline quality and recommendation for use. BMC health services research. 2018;18(1):143. 


\section{Appendix 1}

\section{Overview of AGREE-HS items}

\begin{tabular}{|c|c|c|}
\hline AGREE-HS Item & Description & Criteria \\
\hline Topic & $\begin{array}{l}\text { Description of the health } \\
\text { system challenge, the } \\
\text { causes of the challenge } \\
\text { and the priority accorded } \\
\text { to it, and relevance of the } \\
\text { guidance. }\end{array}$ & $\begin{array}{l}\text { - The health system challenge is clearly described (i.e., the nature of the } \\
\text { challenge; the } \\
\text { magnitude, frequency or intensity of the challenge; the populations } \\
\text { affected). } \\
\text { - The causes of the health system challenge are clearly described. } \\
\text { - The health system challenge is described in terms of its level of priority } \\
\text { in the targeted health } \\
\text { system and the affected population; arguments to support the priority } \\
\text { classification are provided. } \\
\text { - The guidance is relevant to (i.e., timely in relation to when decisions } \\
\text { will be made), and } \\
\text { appropriate for, the health system challenge, the system or sub-system } \\
\text { needs, the target population(s), and the setting(s) in which they will } \\
\text { operate. }\end{array}$ \\
\hline Participants & $\begin{array}{l}\text { Composition of the health } \\
\text { systems guidance } \\
\text { development team and the } \\
\text { management of competing } \\
\text { interests and funder } \\
\text { influence. }\end{array}$ & $\begin{array}{l}\text { - The health systems guidance development team includes members } \\
\text { who have an interest or } \\
\text { stake in the recommendations (e.g., decision makers, program } \\
\text { managers, operational leaders, } \\
\text { consumers and members of the public). } \\
\text { - The health systems guidance development team is multidisciplinary } \\
\text { (e.g., political scientists, } \\
\text { economists, epidemiologists, methodologists). } \\
\text { - The health systems guidance development team is multi-sectoral (e.g., } \\
\text { primary care, public } \\
\text { health and, if appropriate to the challenge, finance and housing). } \\
\text { - Competing interests of the health systems guidance development } \\
\text { team members (e.g., } \\
\text { financial, professional), and the strategies used to identify and manage } \\
\text { them, are clearly described. } \\
\text { - Precautions have been taken to avoid or to minimize the influence of a } \\
\text { funding agency. }\end{array}$ \\
\hline Method & $\begin{array}{l}\text { Use of systematic methods } \\
\text { and transparency in } \\
\text { reporting; the use of the } \\
\text { best available and up-to- } \\
\text { date evidence; the } \\
\text { consideration of } \\
\text { effectiveness and cost- } \\
\text { effectiveness of the } \\
\text { potential options; and the } \\
\text { weighting of benefits and } \\
\text { harms in the guidance } \\
\text { document. }\end{array}$ & $\begin{array}{l}\text { - Systematic and transparent methods were used to identify and review } \\
\text { the evidence (e.g., } \\
\text { integrated review, scoping review, review of the grey literature, } \\
\text { systematic review). } \\
\text { - The best available and most contextually relevant evidence was } \\
\text { considered. } \\
\text { - The evidence base is current. } \\
\text { - Evidence of effectiveness of the potential options is clearly described, } \\
\text { including descriptions of } \\
\text { the contexts in which the options were tested. } \\
\text { - Evidence of cost and cost-effectiveness of the potential options is } \\
\text { described. } \\
\text { - The weighting of the benefits and harms of the potential options is } \\
\text { described. } \\
\text { - There is a link between the recommendations and evidence. } \\
\text { - The rationale behind the recommendations is clear. } \\
\text { - Systematic and transparent methods were used to agree upon the } \\
\text { final recommendations } \\
\text { (e.g., informal or formal consensus, Delphi method, nominal group } \\
\text { methods). }\end{array}$ \\
\hline
\end{tabular}




\begin{tabular}{|c|c|c|}
\hline Recommendations & $\begin{array}{l}\text { Outcomes orientation and } \\
\text { comprehensiveness of the } \\
\text { guidance; the ethical } \\
\text { and equity considerations } \\
\text { drawn upon in its } \\
\text { development; the details } \\
\text { for its operationalization; } \\
\text { the sociocultural and } \\
\text { political alignment of the } \\
\text { guidance; and the updating } \\
\text { plan. }\end{array}$ & $\begin{array}{l}\text { The anticipated outcomes of implementing the recommendations are } \\
\text { clearly described } \\
\text { (including indicators, performance thresholds or targets, and standards } \\
\text { to measure them). } \\
\text { - The recommendations are comprehensive and provide direction to all } \\
\text { relevant health system } \\
\text { levels (e.g., national, provincial/state), subsystems (e.g., cancer, mental } \\
\text { health) and sectors } \\
\text { (e.g., primary care, public health). } \\
\text { - The ethical principles used to develop the recommendations are } \\
\text { described. } \\
\text { - The recommendations promote equity among the target population } \\
\text { (e.g., in terms of age, sex, } \\
\text { gender, culture, religion, race, sexual orientation). } \\
\text { - The recommendations' acceptability to, and alignment with, } \\
\text { sociocultural and political } \\
\text { interests were considered. } \\
\text { - The recommendations are easily identifiable, clear, and succinct. } \\
\text { - The recommendations are actionable and are sufficiently detailed to } \\
\text { be operationalized. } \\
\text { - A plan for updating the recommendations is described. }\end{array}$ \\
\hline Implementability & $\begin{array}{l}\text { Barriers and enablers to } \\
\text { implementing the } \\
\text { recommendations; the cost } \\
\text { and resource } \\
\text { considerations in } \\
\text { implementing the } \\
\text { recommendations; the } \\
\text { affordability of } \\
\text { implementation and } \\
\text { anticipated sustainability of } \\
\text { outcomes; the flexibility } \\
\text { and transferability of the } \\
\text { guidance; and the } \\
\text { strategies for } \\
\text { disseminating the } \\
\text { guidance, monitoring its } \\
\text { implementation and } \\
\text { evaluating its impact }\end{array}$ & $\begin{array}{l}\text { Barriers and enablers to the implementation of the recommendations } \\
\text { are described, including } \\
\text { factors that are internal (e.g., resources, incentives, administrative } \\
\text { structure) and external (e.g., } \\
\text { legal system, social system, state of the economy, corruption, beliefs) to } \\
\text { the health system. A } \\
\text { plan to mitigate barriers and optimize enablers is included. } \\
\text { - Cost and resource considerations for the recommended actions are } \\
\text { described (e.g., money, } \\
\text { time, infrastructure, equipment, administrative capacity, supplies, } \\
\text { staffing, and training). } \\
\text { - The stakeholders' acceptability of the recommendations is described. } \\
\text { - The affordability of the recommendations, in the context where } \\
\text { implementation will take place, } \\
\text { is described. } \\
\text { - The anticipated sustainability and requirements to maintain long-term } \\
\text { outcomes is described. } \\
\text { - The recommendations are flexible and there is a description of how } \\
\text { they can be adapted or } \\
\text { tailored for specific contexts in which they will be implemented. } \\
\text { - A description of the degree to which the recommendations are } \\
\text { transferable to other similar or } \\
\text { different contexts is provided. } \\
\text { - Strategies for disseminating the health systems guidance are } \\
\text { described. } \\
\text { - Strategies for assessing the implementation process and the impact of } \\
\text { the recommendations } \\
\text { are described. }\end{array}$ \\
\hline
\end{tabular}

